\title{
La corrosion par la mer du matériel des usines marémotrices
}

\section{Sea water corrosion of tidal power station equipment.}

\author{
PAR R. RATH ET G. SURREL
}

I. - Lutre conthe la corrosion.

Exposé des méthodes de protection : revêtements et peintures, protection cathodique, choix des métaux résistant plus ou moins à la corrosion. Réalisation de groupes expérimentaux.

II. - Essais effectués a Saint-ServaN.

Définition du phénomène électrochimique de la corrosion, ses causes.

Programme des essais et moyens mis en cuvre. Description des essais effectués avec diverses sortes de repêtements.

Essais relatifs aux métaux résistants, isolés ou en association.

Etude des courbes de polarisation en fonction de la vitesse de déplacement de l'électrolyte.
1. The Struggle aganst Corroston.

Discussion on the methods of protection: Plating and paint coating. Cathodic protection. Choice of metals with anti-corrosive properties. Designing of experimental units.

2. Tests carried ovt at Salnt-Senvax.

Definition of the electro-chemical phenomena of corrosion and its causes.

Test programme and practical application.

Description of the tests carried out with various types of covering.

Tests on anti-corrosive metals, both on their own and in association.

Investigation of polarisation curves in terms of the displacement speed of the electrolyte.

\section{LA LUTTE CONTRE LA CORROSION}

\author{
PAR R. RATH \\ PRELSIDENT DU \& GROUPE CORROSION 》 E.D.F. - S.E.U.H.
}

Les problèmes relatifs au choix des matériaux et à leur protection contre la corrosion et la salissure figurent en bonne place parmi ceux que pose la réalisation industrielle d'une usine marémotrice.

Le «Groupe Corrosion» s'est constitué le 12 juillet 1955 . Il comprend des représentants :

- de la Société d'Etudes pour l'Utilisation des Marées (S.E.U.M.);

- de la Région d'Equipement Hydraulique $\mathrm{N}^{\circ} 8$ (R.E.H.8.);

- de la Production Hydraulique;
- du Laboratoire Central de la Production Thermique;

- du Service de la Construction du Matériel;

- des Etudes et Recherches Gaz de France;

et s'est fixé pour objectif l'établissement d'une doctrine sur les dispositions à prendre pour protéger contre la corrosion et les salissures les diverses structures de l'Usine Marémotrice de la Rance.

La présente note fait le point des études en ce qui concerne les revêtements et peintures, la protection cathodique et le choix des métaux. 


\section{REVETEMENTS ET PEINTURES}

Il est rare qu'un revêtement soit une simple barrière destinée à séparer une structure d'un milieu agressif : c'est en général un système chimique, doué de réactivité.

Il en résulte immédiatement qu'une protection ne doit être choisie qu'après essai sur les lieux d'emploi dans des conditions aussi proches que possible des conditions du service réel qui lui sera demandé.

Pour aboutir rapidement dans le choix qui nous occupe, il a paru nécessaire de consulter les fournisseurs de peintures les plus renommés, d'enquèter auprès des grandes Administrations, des Chantiers maritimes, des Ports, des Centres d'Etudes français et étrangers spécialisés dans la protection des ouvrages à la mer; enfin de procéder à un certain nombre d'essais sur quelques peintures spécialement vantées par les fournisseurs ou déjà connues par ailleurs.

Une bonne protection contre la corrosion exige une excellente préparation des surfaces par décapage ou sablage et le dépôt d'un revêtement d'épaisseur étudiée.

La mise en peinture doit se dérouler suivant les spécifications du «système » choisi :

Décapage ou sablage de la surface, apprêt de celle-ci par un enduit phosphaté on, mieux, par un « wash-primer 》 constilué d'une solution d'acide phosphorique, de butyral polyvinylique et de chromate de zinc, enfin dépôt de plusieurs couches de peinture anticorrosive et, éventuellement, d'une couche de peinture antisalissure.
Les peintures anticorrosives ont été systématiquement étudićes depuis 1939 par la British Iron and Steel Research Association qui a mis au point les formules actuellement utilisées par l'Amirauté et la Mariné marchande britanniques. Les pigments sont des mélanges de sulfate basique de plomb, de carbonate de plomb, de sulfate de baryte naturel et d'un résidu de bauxite obtenu après extraction de l'aluminium. Dans une formule plus récente, le carbonate de plomb est remplacé par la poudre d'alumininm. Les liants utilisés sont des mélanges de standolie et de vernis phénol-formol.

Aux Etats-Unis, les recherches du Bureau of Ships ont abouti à des formules différentes faisant un large usage de la poudre de zinc, de l'oxyde et du chromate de zinc, dans des liants à base de résines phénoliques, glycérophtaliques ou encore vinyliques.

En France, les recherches systématiques ont été peu nombreuses, mais ont mis en vedette les peintures à la poudre de zine pour les travaux à la mer. Par contre, les peintures vinyliques ne font encore qu'une timide apparition.

Les peintures antisalissures ont été très étudiées aux Etats-Unis. Leur durée de vie est limitée à quelques mois, car elles ne sont actives que dans la mesure où les pigments toxiques - sels de cuivre, de mercure, etc. - se dissolvent dans l'eau, par simple diffusion au travers de la pellicule de liant ou par perte du liant lui-même. Leur emploi suppose donc des carénages fréquents.

\section{PROTECTION CATHODIQUE}

On sait que la protection cathodique d'une structure immergée consiste à la porter à un potentiel électronégatif par rapport au liquide d'immersion.

La protection cathodique a déjà fait ses preuves dans le domaine des constructions maritimes; depuis 1948 la Marine Royale Canadienne a équipé plus de 50 navires avec des anodes en magnésium et a fait des essais concluants avec des anodes en fer ou graphite; à Dunkerque, après deux ans d'essais, il a été décidé d'installer une protection sur les bajoyers de l'écluse Trystram.

L'application de la protection cathodique à l'usine de la Rance nécessita l'examen préalable de quelques points particuliers :

- Influence de la protection cathodique sur la tenue des peintures;

- Influence de la vitesse de l'eau sur la polarisation du métal;

- Influence de la vitesse de l'eau sur la formation du dépôt calco-magnésien, qu'on observe d'ordinaire sur les surfaces nues protégées cathodiquement;

- Influence de la protection cathodique sur le potentiel electrochimique des structures voisines et non protégées. 


\section{CHOIX DES METAUX}

Les constructeurs utiliseront les aciers ordinaires ou peu alliés dans toute la mesure du possible et réserveront les acier's et alliages inoxydables (laiton) aux cas spéciaux.

Pour faciliter le choix des constructeurs, le Groupe Corrosion a procédé à une enquête auprès des Compagnies de navigation afin de rassembler tous les renseignements possibles sur le problème des hélices de bateau, et il a imaginé une série d'essais à la mer.

Enguête. - Pour les grosses hélices, la Compagnie Générale Transatlantique utilise le laiton 60-40 conforme aux normes de la Marine Nationale; l'Institut de Recherches de Construction Navale confirme que les seuls métaux utilisés sont les laitons HR ou THR. Aux Etats-Unis, la situation est analogue et, d'après le Ministère du Commerce américain, aucun navire de la flotte marchande n'utilise d'hélice en acier inoxydable. En Allemagne, au contraire, il semble qu'on ait été satisfait des aciers inoxydables. Les Suédois l'utilisent de plus en plus et y trouvent de nombreux avantages.

Mais les aciers inoxydables présentent un débit de court-circuit notable en présence d'acier ordinaire. Le débit de court-circuit laiton-acier ordinaire est plus important encore. Il en résulte que, dans un cas comme dans l'autre, des précautions devront être prises pour éviter la corrosion provoquée par le contact de métaux différents, et seuls des essais à la mer permettront de mettre au point les dispositifs appropriés.

\section{REALISATION DES GROUPES ÉCLUSE}

Pour fixer les idées, rappelons la construction schématique d'un groupe :

a) La calotte amont et ses bras d'ancrage dans le béton du pertuis, non démontable.

Matériaux possibles : acier inoxydable, acier ordinaire laminé, acier coulé.

b) Le groupe bulbe proprement dit, son conduit ainsi que le distributeur et l'avant-distributeur.

Matériaux possibles : conduit en acier laminé, bulbe en acier laminé et acier coulé.

c) Roue et ceinture de roue (cette dernière fait partie du conduit).

Matériaux possibles : pales en acier inoxydable ou en bronze; ceinture de roue et moyeu : acier inoxydable, en acier ordinaire plaqué bronze ou plaqué acier inoxydable.

L'ensemble des parties $b$ et $c$ forme le monobloc démontable.

d) Ogive : elle comprend deux parties : l'une est démontable avec le monobloc, l'autre, qui constitue l'extrémité du groupe, est amovible.

Afin d'obtenir sur les problèmes de corrosion le maximum de renseignements, les deux groupes qui seront installés dans l'écluse de SaintMalo sont prévus :

- avec des assemblages d'acier moulé et d'acier coulé,

-- avec des états de surface différents,

- avec peinture totale ou partielle (ou nulle),

- avec protection cathodique totale ou partielle,

- avec peinture et protection cathodique.

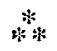

D'importantes séries d'essais nous ont paru nécessaires sur les principaux points :

- Revêtements el peintures,

-C Choix des métaux.

Ils ont été entrepris à notre demande par le S.E.U.M. de Saint-Malo, sur place, dans les conditions mêmes de la future usine marémotrice. M. Surrel vous en rendra compte.

Les essais sur la protection cathodique sont du domaine du Gaz de France. L'équipe de M. Changarnier s'en est occupce et ses travaux seront connus sous peu. 


\title{
DEUXIEME PARTIE
}

\section{ESSAIS A SAINT-SERVAN}

\author{
PAR G. SURREL
}

INGÉNTEUR CHEF DE T'ÉCHET.ON DE SAINT-MALO DU S.E.U.M.

\section{1. - INTRODUCTION. - LE PHÉNOMENE ÉLECTROCHIMIQUE DE LA CORROSION}

\section{1. - La corrosion, dans le cas qui nous intéresse, est un phénomène électro- chimique.}

C'est le savant suisse Auguste de Larive, étudiant l'attaque du zinc impur par l'acide sulfurique, qui l'a démontré poux la première fois au cours du xix ${ }^{0}$ siècle. Plus récemment, Evans [1], avec l'aide des collaborateurs de son Ecole, a démontré l'équivalence quantitative des phénomènes électriques et des phénomènes de corrosion. Ces derniers chercheurs utilisaient des plaques métalliques immergées dans un électrolyte, présentant des figures de corrosion bien reproductibles entre elles: on distinguait nettement une auréole cathodique et une zone anodique.

Evans découpe une de ces plaques en suivant la frontière entre la zone cathodique et la zone anodique, isole électriquement ces deux zones et les réunit par un circuit électrique comportant un galvanomètre et une source permettant de faire varier la différence de potentiel entre cha-

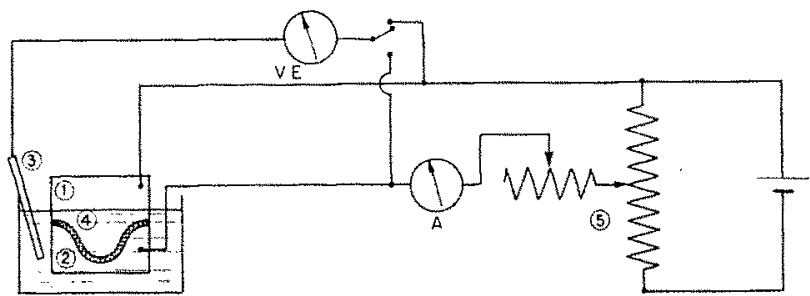

Fic. 1. - Sehéma du dispositif Evans pour la mise el évidence de Ia nature électrochimique des phénoménes de comosion.

(1) Plaque decoupée: partie anodique.

(2) Plaque déconpée : partie cathodique.

(3) Electrode impolarisable pour la mesure des potentiels.

(4) Séparation électriquement isolée des deux parties de la plaque épronvette.

(5) Dispositif potentiométrique de réglage des potentiels de l'éprouvette. cune des parties isolées et l'électrolyte (fig. 1).

Il trace, en fonction de ces différences de potentiel mesurées par l'intermédiaire d'électrodes impolarisables, l'intensité débitée (fig. 2).

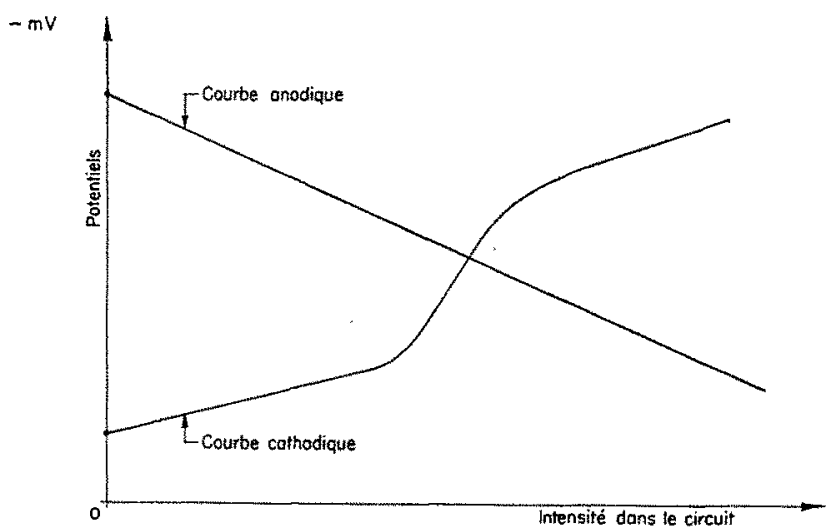

FIG. 2. - Type de diagramme d'Evans.

Muni de cette loi * Intensité en fonction de la différence de potentiel $»$, il étudie une plaque non découpée, mesure en permanence les potentiels en chacun des points, en déduit, par son étalonnage précédent, l'intensité du courant circulant; au bout d'un certain temps, il pèse sa plaque-éprouvette et n'a pas de peine à démontrer que le poids de métal perdu est donné par la loi de Faraday, dans laquelle on prend comme intensité l'intensité lue sur sa courbe d'étaIonnage.

\section{2. - Les causes de la corrosion.}

Dans un électrolyte homogène, un métal homogène ne se corroderait théoriquement pas, mais les causes d'hétérogénéité étant nombreuses, des piles locales prennent naissance. Il existe des possibilités d'hétérogénéité géométriques, physiques ou chimiques : 
- sur la surface du métal,

- sur le revêtement de cette surface,

- dans la masse même du métal,

- dans l'électrolyte (en particulier aération différentielle),

causées par les conditions extérieures telles que champs divers : irradiation, eclairement, température, facteurs biologiques, vitesse de l'électrolyte.

\section{3. - Eléments nécessaires de la corrosion électrochimique.}

Dans toutes les études relatives aux phénomènes de corrosion, on doit se souvenir qu'il faut trouver :

- une zone anodique,

- une zone cathodique,

- une liaison électronique entre ces deux zones,

-- une liaison ionique entre ces deux zones.

\section{2. - PROGRAMME ET MOYENS D'ESSAIS}

Examinons maintenant le programme des essais confiés au laboratoire "in situ » [6] du Service d'Etudes des Usines Marémotrices (S.E.U.M.) à Saint-Servan par le groupe d'étude * corrosion des usines marémotrices * présidé par M. Rath. Nous énumérerons ensuite los moyens mis à notre disposition pour mener à bien ces essais.

\section{1. - Programme.}

Il prévoyait les études :

- des revêtements protecteurs de l'acier contre la corrosion;

- du choix des métaux nobles et de leur association.

Il y aura forcément, dans les machines marémotrices, de nombreuses structures en acier ordinaire, mais les hélices et les ceintures dans les zones de grande vitesse seront probablement en métal noble : acier inoxydable, ou bronze. Nos expériences sur les hélices de bateaux montrent qu'on ne peut faire ces pales et ces ceintures en acier ordinaire revètu, car nous n'avons pas trouvé jusqu'à présent de revêtement plastique donnant satisfaction. Il convient de signaler cependant qu'un essai actuellement en cours, avec un plastique francais, semble ouvrir des espoirs dans cette voie.

Si ces espoirs se confirment, il sera peut être possible d'envisager avec prudence l'emploi de ces matières plastiques, sous forme massive, pour les pales de rone on les ceintures.

\section{2. - Moyens offerts.}

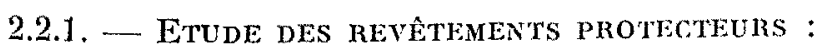

Nous avons utilisé le C.C.C.C. existant (Centre de Contrôle de Corrosion de Cézembre. Directeur : M. Delville) et nous avons créé deux stations d'essais en Rance. a) La station d'essais aux embruns et à l'air salin (C.C.C.C.) se trouve dans l'île de Cézembre sur une plate-forme rocheuse, à une cote de quelques mètres au-dessus des plus hautes caux, exposée au N.O., e'est-à-dire aux vents dominants et bien dégagée pour l'exposition au soleil, à la pluie et aux embruns.

Le C.C.C.C. appartient à M. Delville, qui a bien voulu accepter de se charger de nos essais et d'en suivre le déronlement.

b) La station d'essai au marnage a été créée de toutes pièces par le S.E.U.M.; les plaques éprouvettes sont exposées sur une estacade de bois, sur la Pointe de la Briantais (c'est-à-dire sur l'une des culées du futur barrage de la Rance), à la cote +6 , niveau moyen des marées; elles sont, la moitic du temps dans l'eau et, pour l'autre moitié, émergées.

c) Pour l'étude en immersion prolongée, nous avons établi, à $50 \mathrm{~m}$ au sud de l'île de Chalibert, une sapine. Sont exposés à l'immersion des plaques et des tubes verticaux [12] qui permettent d'étudier les effets de l'aération différentielle. Cette sapine comporte un thermometre enregistreur :

\subsection{2. - ETUDE DU CHOIX ET DE L'ASSOCHATION DES MÉTAUX :}

\section{a) Machine G.E.M. :}

Nous disposons de deux groupes bulbes Rance en modèle réduit, dits groupes G.E.M. (c'est-ädire Groupe d'Etude des Matériaux).

Un ancien blockauss allemand tient lieu de citerne; un groupe hulbe, fonctionnant en pompe, refoule l'eau de ce blockhauss dans l'autre groupe fonctionnant en turbine, puis l'eau est restituée au blockauss.

Les groupes G.E.M. reproduisent le choix des métaux et le mode de revêtement prévu pour les groupes-bulbes en Rance. 
b) Station d'étude de la polarisation des métaux en eau vive:

Pour l'étude de l'association des métaux, nous avons mis au point, en collaboration avec l'équipe Gaz de France, dirigée par M. Changarnier plus

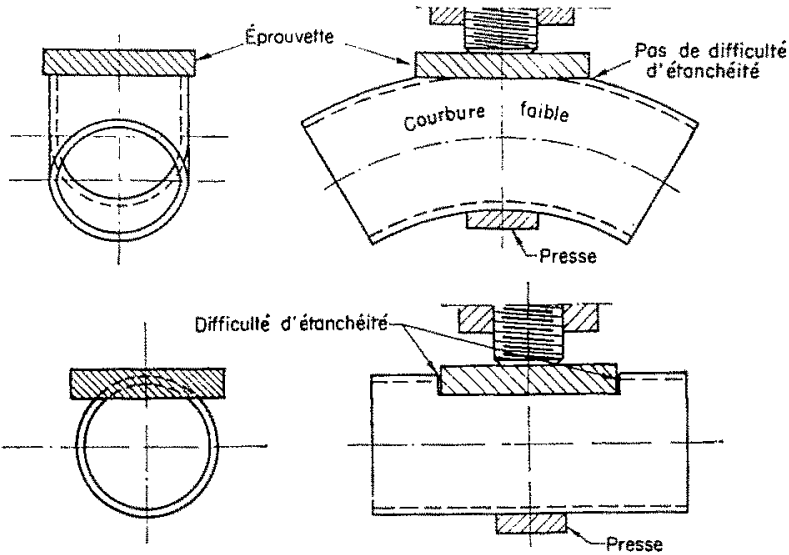

Frc. 3.

a) Notre dispositif

Scliéma de l'appareil pour le tracé des courbes de polarisation en eau vive

b) Dispositif américain (R.B. TEEL [7] spécialement chargé de ces études d'électro-chimie de la corrosion, un dispositif permettant de tracer les courbes de polarisation des différents métaux envisagés, en présence d'eau de mer vivante et en mouvement (fig. 3 et 4 ).

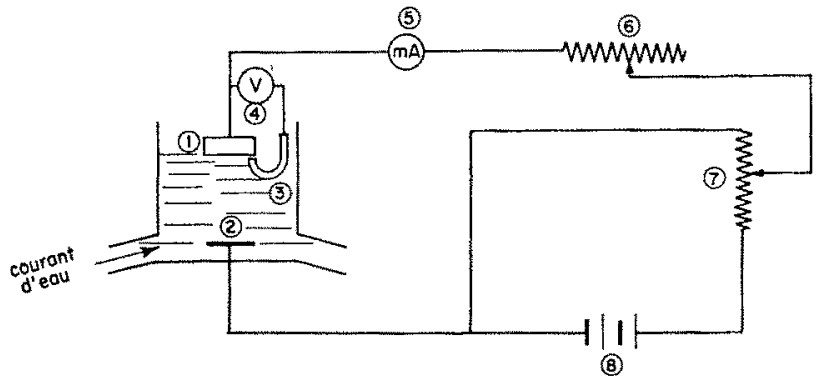

FIg. 4. - Schéma électrique du dispositif ulilisé pour le tracé des courbes de polarisation en eau vive.

(1) Electrode éprouvette.

(2) Electrode d'introduction du courant de polarisation.

(3) Electrode impolarisable au calomel pour mesure du potentiel.

(4) Voltmètre électronique de précision.

(5) Milliampèremètre de précision.

(6) Rhéostat Vermier de l'intensité.

(7) Potentiomètre de dégrossissage de l'intensité.

(8) Batterie.

\section{3. - ESSAIS EFFECTUES SUR LES REVÊTEMENTS ET INTERPRETATION DE CES ESSAIS}

\section{1. - Campagne 1954-1955.}

\subsection{1. - Nature des REVÊtements essayês ET DATES D'EXPOSITIONS :}

Nous avons procédé à une sérieuse étude des revêtements au C.C.C.C. et à un début d'essais de revêtements en immersion totale.

Mentionnons tout de suite qu'aucun des produits essayés en immersion n'a donné satisfaction cette année-là.

Les dates d'exposition et la nature des revêtements essayés figurent sur le tableau I.

\section{TABLEAU I}

Essais efrectués aU C.C.C.C.

(Exposition à lair salin et aux embruns)

\section{CaMPagne 1954-1955}

1) Dates d'exposition :

- 15 systèmes de peintures furent exposés dès le début de mai 1955 ;

- 9 nouveaux systèmes le 13 août 1955 ;

- 8 nouveaux systèmes le 19 novembre 1955.

2) Nature et nombre de revêtements essayés :

- Bitumineux ........... 1
- Oléosynthétiques . . ...... 6

— Glycérophtaliques ....... 6

- Zinc ............... 2

- Caoutchoue synthétique.... 2

- Caoutchouc chloré........ 3

- Epikote .............. 3

- Vinylique ............ 4 (deux fournisseurs, mais un essai de trois techniques d'application différentes pour l'un d'eux)

- Isocyanates . . . . . . . . . . 1

- Cyanamides ........... 1

- Impossible à déterminer... 3

ToTal..... 32

Les conditions d'application avaient été très soigneuses et les mêmes pour tous les systèmes : sablage humide, application de la première couche dans les trois heures, application des autres couches strictement conforme aux indications des fournisseurs.

\section{1:2. - Résultats [8] :}

La corrosion a été plus rapide que nous ne le pensions et, au bout d'un an, nous avancions un classement des éprouvettes. Sur les 32 systèmes 
essayés, nous avons conservé en exposition 7 systèmes, dont 3 vinyliques.

Les peintures en zinc, qui s'étaient bien comportées initialement, ont assez brutalement failli vers la fin de l'essai [9].

Les peintures vinyliques se sont montrées généralement supérieures.

Les éprouvettes ont péri par apparition de points de rouille par cloquage, par craquellements, par décollement ou par farinage.

\section{2. - Campagne 1955-1956 :}

Elle fut plus complète.

Elle comportait, en plus des essais à l'air marin et aux embruns, des essais sur l'estacade (immersion-émersion) et des essais sur la sapine (immersion prolongée).

\subsection{1. - NATURE DES REVÊTEMENTS ESSAYÉS ET DATES D'EXPOSITION :}

Nous avons exposé :

- 15 systèmes vinyliques;

- - une dizaine de systèmes au zinc (peintures ou revêtements par projection);

- 1 caoutchouc synthétique.

Le tableau II précise la nature des systèmes et des expositions ainsi que les dates d'exposition.

\subsection{2. - RÉsultat des essais :}

Ces résultats sont extraits des rapports de surveillance dressés par M. Delville [8] pour le C.C.C.C. et par M. Romanovsky [13] qui a bien voulu collaborer avec nous à titre de Conseil, pour les stations de la Rance, c'est-à-dire en immersion-émersion et immersion prolongée.

\section{A. Classement des stations \\ par agressivité :}

Nos trois stations se classent ainsi, par ordre d'agressivité décroissante :

- Rance, Chalibert : immersion prolongée.

- Rance, Estacade : immersion-émersion.

- C.C.C.C. : embruns et air salin.

\section{B. - Concordance des résultats :}

Les résultats de ces trois stations sont concordants et concordent également avec les essais de résistance des revêtements à la protection cathodique entrepris, indépendamment, par M. Changarnier [14] en eau de mer artificielle dans son laboratoire de Paris.

Sur 15 systèmes de peintures vinyliques essayés dans les stations Cézembre et Rance, 4 ont été classés bons dans les deux, 8 ont été classés mauvais et 3 seulement n'ont pas donné lieu à jugement concordant.

\section{c. - Les revêtements au zinc:}

Les revêtements au zinc ont une adhérence médiocre, les cireuits électroniques fer-zinc et zinc-zine se coupent; le zinc s'épuise : ils n'ont peut-être pas dit leur dernier mot [18].

\section{D. - Les revêtements vinyliques :}

a) Phénomínes observés :

Cloques. - Les peintures vinyliques cloquent presque toutes; ce défaut est d'ailleurs signalé par les Américains [16]. La peau des cloques comprend toutes les couches de peinture, wash primer compris dans certains cas, non compris dans d'autres.

TABLEAU II

Installation des ÉProvvettes aUx CONDitrons de CORRosion. - Campagne 1956

\begin{tabular}{|c|c|c|c|c|c|c|c|c|c|c|c|c|}
\hline \multirow[b]{2}{*}{ SYSTĖMES ESSAYÉS } & \multicolumn{3}{|c|}{ Cézembre } & \multicolumn{3}{|c|}{ Estacade (MARNage) } & \multicolumn{3}{|c|}{ IMMERSION (PLAQUES) } & \multicolumn{3}{|c|}{ IMMERSION (TUBES) } \\
\hline & 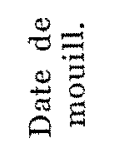 & $\underbrace{0}_{0}$ & 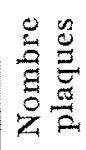 & 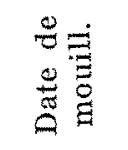 & 泀葛 & 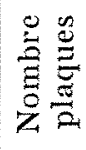 &  & 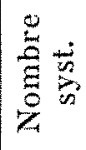 & 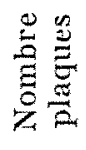 & 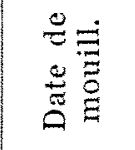 & 总蒡 & 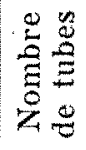 \\
\hline Vinyliques & & & & & & & & & & & & \\
\hline $\begin{array}{l}\text { Sans protection cathodique : } \\
\text { Finition inerte } \ldots\end{array}$ & 11.5 .56 & 15 & 45 & 11.5 .56 & 10 & 15 & 20.5 .56 & 13 & 15 & 10.10 .56 & 5 & 5 \\
\hline Finition antifouling... & - & - & - & 11.5 .56 & 3 & 3 & 20.5 .56 & 3 & 4 & 19.10 .56 & 7 & 7 \\
\hline Avec protection cathodique : & & & - & 11556 & 10 & 10 & 20.5 .56 & 8 & 8 & 19.11 .50 & 5 & 5 \\
\hline Finition antifouling................. & - & - & - & 11.5 .56 & 3 & 3 & 20.5 .56 & 6 & 6 & 19.11 .56 & 7 & 7 \\
\hline $\begin{array}{l}\text { Zinc ou autres } \\
\text { Sans protection cathodique. }\end{array}$ & 11.5 .56 & 7 & 21 & $\begin{array}{l}11.5 .56 \\
11.556\end{array}$ & $\begin{array}{r}13 \\
7\end{array}$ & $\begin{array}{r}14 \\
7\end{array}$ & $\begin{array}{l}20.5 .56 \\
20.5 .56\end{array}$ & $\begin{array}{l}12 \\
10\end{array}$ & $\begin{array}{l}14 \\
10\end{array}$ & $\begin{array}{l}19.10 .56 \\
19.11 .56\end{array}$ & $\begin{array}{l}3 \\
3\end{array}$ & $\begin{array}{l}3 \\
3\end{array}$ \\
\hline Avec protection cathodique. & - & - & - & 11.5 .56 & $\frac{7}{46}$ & $\frac{7}{52}$ & 20.5 .50 & 52 & 57 & & 30 & 30 \\
\hline
\end{tabular}


Perte d'adhérence. - Un article récent paru dans la revue Corrosion signale que les difficultés d'adhérence tiendraient, non au collage de la première couche, dite « wash primer », sur le métal lui-mème, mais au collage des couches ultérieures sur ce wash primer.

Le cloquage s'accompagne d'une perte d'adhérence caractéristique au roisinage, mais jusqu'assez loin, des cloques (fig. 5).

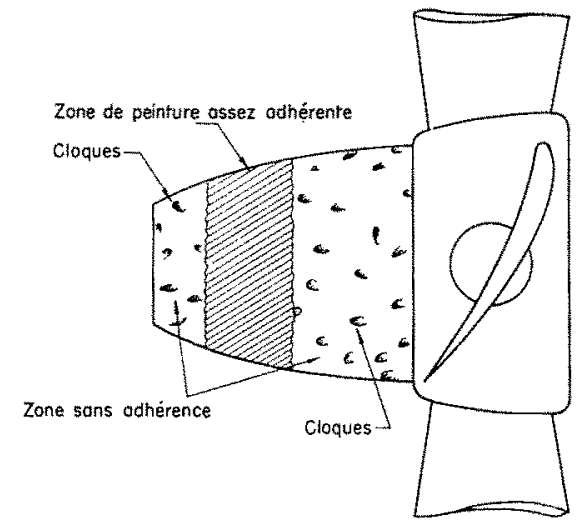

Fid. 5, - Machine G.E.M. - Ogive.

Topographic des eloques et de ladherenee de la peinture.

Liquide des cioques. -- Les cloques sont remplies d'un liquide dont le pH est de l'ordre de 13. Nous pensons donc que ces cloques sont cathodiques [107. Si on les crève, elles donnent lieu à des plages anodiques qui se rouillent.

Influence de la finition. - De deux systèmes vinyliques du même fournisseur terminès, l'un par une finition à pigments inertes, et l'autre par une finition antifouling, le moins cloqué est celui comportant la finition antifouling.

Influence de la vitesse du fluide. - Le phénomène de circulation d'eau rapide favorise le cloquage et la perte d'adhérence. Nous l'avons nettement constaté avec les G.E.M.

Influence de l'épaisseur du revêtement. - Les systemes vinyliques minces paraissent donner de meilleurs résultats au cloquage; un boulon observé par M. Romanovsky s'est bien conservé pendant près d'un an d'immersion avec une seule couche de primer vinylique.

De même, une directrice de la machine G.E.M., peinte accidentellement d'une seule couche de wash primer, a relativement mieux résisté que le reste de la peinture.

Gonflage des revétements à l'immersion.-Les peintures vinyliques gonflent à l'immersion; les épaisseurs ont augmenté en cours d'immersion.

Résistance électrique des cloques. - M. Changarnier a observé que la résistance électrique des éprouvettes immergées reste grande jusqu'à l'apparition de la première cloque. Elle s'effondre lors de cette apparition.

\section{b) Essai d'interprétation de ces faits :}

Perméabilité des peintures. - Toute peinture est perméable, en particulier à l'eau, à l'oxygène et à l'hydrogène. Nous avons vu qu'il s'établit sur toute surface métallique peinte, par suite des hétérogénéités diverses, une topographie des potentiels : certaines parties sont cathodiques, d'autres anodiques.

Explication de Mayne [17]. - Les cloques cathodiques seraient, d'après Mayne, un phénomène complexe d'électrosmose, dû̀ au fait que le film peut être considéré comme comportant de grosses molécules polarisées négativement.

Si on inverse la polarité de la conche, les cloques qui étaient cathodiques deviennent anodiques.

Mayne signale qu'un film de peinture immergé roit sa résistance électrique diminuer fortement car il s'imbibe d'électrolyte. Mais il a effectué cette expérience, semble-t-il, sur un film sans support.

Théorie de la couche d'adhérence. - Nous ne pensons pas que l'explication électrosmotique de Mayne soit pleinement satisfaisante : on a pu observer des cloques sur des plaques de verre on de matière isolante, où le phénomène électrique doit être peu important.

D'autre part (Changarnier), la résistance électrique des peintures vinyliques reste excellente jusqu'à l'apparition de la première cloque. Le film de la cloque n'ayant pas de support, nous sommes alors seulement ramenés au cas étudié par Mayne.

Nous suggérons l'explication suivante: le fait que le film soit collé sur un métal introduit une propriété que ne possède pas le film seul : la conche d'adhérence ou couche de collage; tant que cette couche de collage existe, il ne semble pas que l'eau puisse atteindre le métal de façon massive. Par contre, cette couche est fragile, elle peut être endommagée par les phénomènes d'écoulement d'eau (machines G.E.M. et Dechanx [10]), par chocs on dragage (cas des pétroliers américains peints en peinture vinylique et qui raclent le fond du canal de Suez [16]), par effets électrolytiques (décollement lors des essais Changarnier [14]), gonflement de la couche de finition (efforts de décollement [5] fig. 6) soit au milieu de la plaque, soit de préférence au bord de la plaque.

Une fois la couche de collage rompue, l'eau peut imbiber la totalité du film de peinture et arriver en force au métal, faisant s'écrouler la résistance électrique de l'ensemble.

Alors, seulement, le développement de la clo- 
que amorcé est favorisé par endosmose et électrosmose.

La meilleure tenue des peintures antifouling pourrait donc s'expliquer par le fait que les caractéristiques mécaniques et les caractéris-

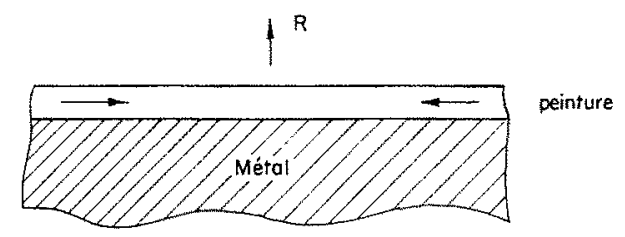

a) Décolloge de la peinture ou centre de la plaque

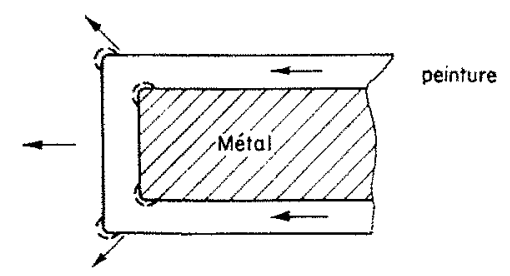

b) Décollage de 10 peinture aux bouts de la plaque

Fia. 6. - Effets de gonflement de la peinture détruisant la « couche de collage». tiques de gonflement de la couche de finition antifouling ne seraient pas les mèmes que celles des couches de finition inertes: n'oublions pas qu'elles contiennent des ions cuivre positifs qui, peut-être, rendent le phénomène de l'électrosmose moins agressif.

\section{c) Efficacité contre les salissures:}

L'eau de la Rance est un milieu tris habité. II faut s'attendre, en mars et en septembre, à une prolifération d'organismes sur les surfaces exposées.

Les peintures au zinc semblent présenter de faibles propriétés antifouling.

Les peintures vinyliques à finition inerte sont mauvaises. Les peintures vinyliques à finition antifouling se comportent bien : sur les cinq peintures antifouling peu cloquées - ou non cloquées - subsistant, dans nos stations, de l'essai 1956, deux sont exemptes de salissures, les trois autres présentent quelques algues. Nous avons déjà signalé que ces qualités antifouling s'obtiennent sans altérer les qualités de résistance à la corrosion : la littérature américaine nous avait mal préparé à ce résultat.

\section{4. - CHOIX DES METAUX - LEUR ASSOCIATION}

\section{1. - Résultats des essais sur groupe G.E.M.}

\subsection{1. - EN TURBINE :}

Nous avons essayé sur la turbine deux aciers nobles : deux pales étaient en acier à $23 \% \mathrm{Cr}$, $8 \% \mathrm{Ni}, 2 \%$ Mo. Les deux autres pales étaient en acier Cr $20 \%$ - Ni 7,5\% - Mo 2,5\% Cu 1,5\%.

L'une des deux pales représentatives de chaque acier était polie, l'autre surfacée moins finement; la ceinture était en acier 18/8 et les directrices en acier semi-inoxydable, à $4 \%$ de chrome, $1 \%$ de nickel, $1 \%$ d'aluminium.

\subsection{2. - EN POMPE:}

La ceinture et les pales étaient en acier à $13 \%$ de chrome, $2 \%$ de nickel ( 2 pales polies, 2 autres moins bien surfacées) et les directrices en acier ordinaire. Une pale et la ceinture comportaient des recharges de soudure électrique à l'acier $18 / 8$.

Au bout de 2.200 heures de fonctionnement, l'évolution du système paraissait arrêtée et on pouvait classer les aciers dans l'ordre suivant :

1. - Acier à $23 \%$ de chrome;

2. - Acier à $20 \%$ de chrome;

3. - Acier à $13 \%$ de chrome;
4. - Acier semi-inoxydable à $4 \%$ de chrome;
5. - Acier ordinaire.

L'état de surface des métaux est important: les pales polies présentent une résistance à la corrosion meilleure que les pales usinées.

Par contre, la peinture vinylique n'a pas si bien résisté que dans l'essai en immersion.

Actuellement un deuxième essai est en cours, avec d'autres pales, sur la turbine, en acier voisin du 17/4/4 PH Armeo, et nous allons également changer les pales de la pompe.

\subsubsection{INTERPRÉTATION DE CES RÉSULTA'TS :}

L'interprétation de ces résultats présente quelques difficultés du fait de la complexité de lá machine G.E.M. qui comporte plusieurs métaux, mais en se servant du diagramme de polarisation d'Evans, on doit pouvoir les interproter qualitativement d'abord et même tenter une interprétation quantitative [5].

\section{2. - Etude de l'association des métaux. - Tracé des courbes de polarisation.}

\subsection{1. - LES MESURES ET LES RÉSUltats :}

L'équipe G.D.F. sous la direction de M. Legrand a tracé à Saint-Malo les courbes de polarisation 
anodiques et cathodiques de 9 métaux pour 7 vitesses du courant d'eau.

Les deux diagrammes de la figure 7 rendent
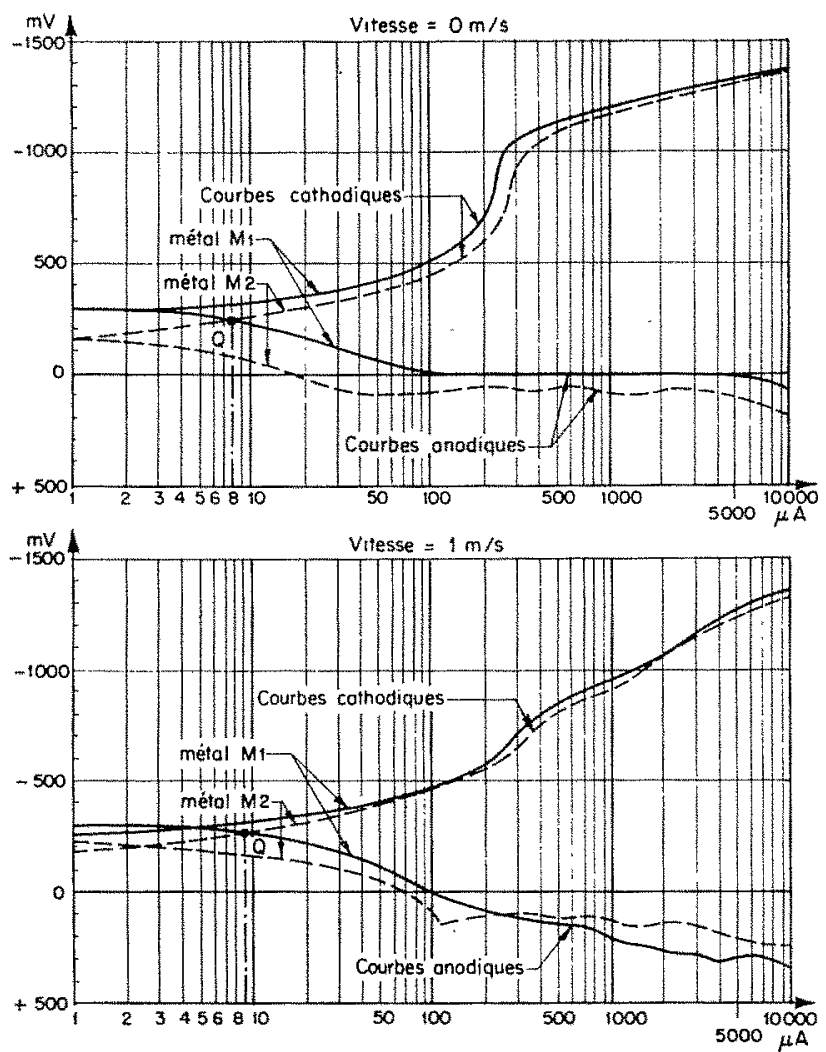

Fig. 7. - Courbes de polarisation de deux métaux $M_{1}$ el $\mathrm{M}_{\mathrm{z}}$, en eau de mer de $\mathrm{pH}$ \# (surface d'électrode: $\left.5 \mathrm{~cm}^{2}\right)$.

$\longrightarrow \mathrm{M}_{1} \quad \ldots-\mathrm{M}_{2}$

compte de la façon dont on peut déterminer l'intensité du courant de corrosion entre deux métaux de nature différente.

On trace, sur le mème diagramme, les courbes cathodique et anodique de chacun d'eux. Le point $Q$ d'intersection de ces deux courbes donne l'intensité du courant du couple galvanique simple formé par ces deux métaux dans le cas, pessimiste, où la résistance du circuit est nulle.

L'équipe Changarnier a résumé les conclusions essentielles de ses travaux dans le tableau III. Les métaux nos 1 et 8 sont compatibles entre eux, car on admet qu'un métal est très stable quand il se consomme à la vitesse de $1 / 10^{8}$ de gramme par $\mathrm{m}^{2}$ et par heure, ce qui correspond à une densité de courant de 10 micro-ampères par $\mathrm{cm}^{2}$ soit, dans notre cas, puisque nos électrodes avaient $5 \mathrm{~cm}^{2}, 50$ microampères pour $5 \mathrm{~cm}^{2}$. Aucun de ces métaux ne donne d'association stable avec le métal 9, qui n'est autre que l'acier ordinaire.
4.2.2. - Quelques ConsÉquences Des PROpRiéTÉS DE POLARISATION DES MÉTAUX :

A) Association métal noble-acier ordinaire peint sans protection cathodique si le revêtement vient à être blessé (fig. 8).

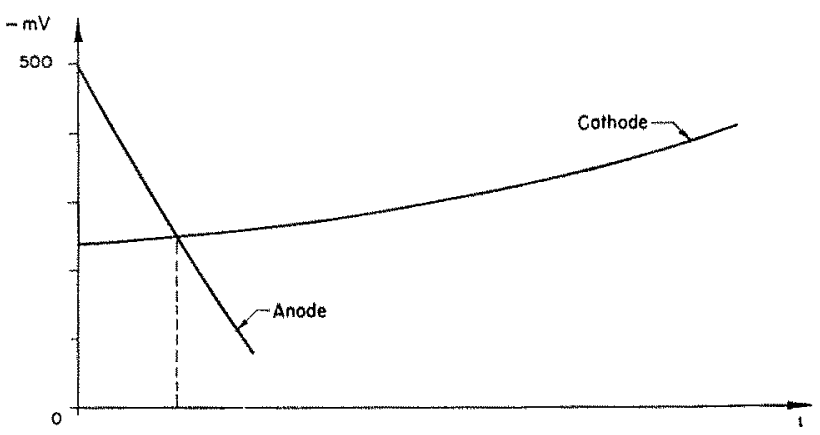

Fic. 8. - Diagramme de polarisation résultant d'une petite anode par blessure du revetement du métal (controle anodique).

On roit que la faible dimension de l'anode ne permet pas une polarisation importante de la cathode et on trouve par conséquent sur l'anode la presque totalité de la tension du couple, done une intensité assez grande localisée sur la petite surface de la blessure, d'où le fameux pitting (corrosion en profondeur) qui peut etre grave s'il n'est pas surveillé.

B) Association métal noble-acier ordinaire non revêtu sous protection cathodique - Protection cathodique minimum.

Examinons cette même association avec protection cathodique acier ordinaire non revêtu (fig. 9).

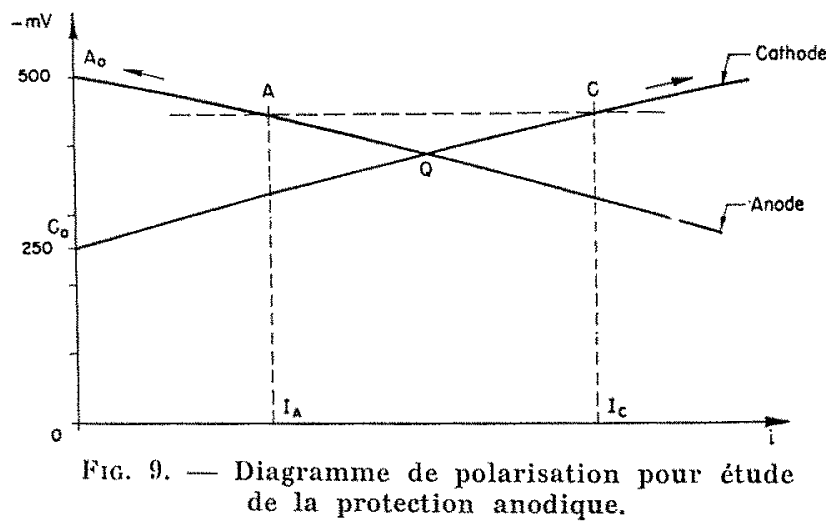

Nous désirons que l'acier ordinaire ne soit pas anode: cette condition idéale de protection cathodique à débit nul du métal le moins noble est appelée "protection cathodique minimum ».

Etudions ce cas particulièrement important. Relions électroniquement par une liaison sans résistance le métal noble et le métal moins noble 
TABLEAU III

Débit maxinum des couples en eau DE mer. - MétauX divers

\begin{tabular}{|c|c|c|c|c|c|c|c|c|c|}
\hline & Métal 1 & Métal 2 & Métal 3 & Métal 4 & Métal 5 & Métal 6 & Métal 7 & Métal 8 & Métal 9 \\
\hline Métal $1 \ldots .$. & 0 & A 5 & C 1 & $\mathrm{C}^{2}$ & A 10 & 0 & 0 & 0 & A 120 \\
\hline Métal $2 \ldots$ & C 5 & 0 & C 3 & C 5 & A 5 & 0 & $\mathrm{C} 2$ & $\mathrm{Ci} 4$ & A 90 \\
\hline Métal $3 \ldots$ & A 1 & A 3 & 0 & C 1 & A 5 & 0 & 0 & 0 & A 75 \\
\hline Métal $4 \ldots$ & A 2 & A 5 & A 1 & 0 & A 6 & A 4 & A 2 & A 1,3 & A 100 \\
\hline Métol 5 . & C 10 & C 5 & C 5 & C 6 & 0 & 0 & C 3 & $\mathrm{C}_{4}$ & A 90 \\
\hline Métal $6 \ldots$ & 0 & 0 & 0 & $\mathrm{C} 4$ & 0 & 0 & C. 2 & $\mathrm{C} 2$ & A 50 \\
\hline Métal $7 \ldots$ & 0 & A 2 & 0 & $\mathrm{C} 2$ & A 3 & A 2 & 0 & $\mathrm{C} 2$ & A 75 \\
\hline Métal $8 \ldots \ldots$ & 0 & A 4 & 0 & C 1,3 & A 4 & A 2 & A 2 & 0 & A 75 \\
\hline Métal 9. & C 120 & C 90 & C 75 & Cit 100 & C 90 & C. 50 & C 75 & C 75 & 0 \\
\hline
\end{tabular}

Surface de $5 \mathrm{~cm}^{2}$, surface égale d'anode et de cathode.

- Les lettres $\mathrm{A}$ ou $\mathrm{C}$ se rapportent à la polarité relative du métal en tête de colonne.

- Les chiffres donnent le débit en $\mu \mathrm{A} / \mathrm{cm}^{2}$ du courant maximum que peut fournir le couple métal colonne/métal ligne, quand ces deux métaux sont équipotentiels (résistance électronique de leur liaison négligeable).

du couple protégé; faisons circuler un courant de sens convenable entre ce couple et une électrode auxiliaire. Ce courant va tendre à dépolariser l'anode et à polariser la cathode (évolution de l'horizontale $\mathrm{AC}$ vers le haut quand le courant de protection croit). Quand l'horizontale $A C$ passe par $\mathrm{AO}$, on est dans les conditions de protection cathodique minimum.

Robert Popp [4], ingénieur américain, a résumé très bien les conditions de protection cathodique minimum.

Nous citons ses conclusions :

1. Les potentiels de lanode et de la cathode sont égaux aux potentiels statiques de l'anode dans le cas d'un couple galvanique simple sans résistance (ce cas, idéal, donnant le courant de corrosion maximum).

2. La polarisation de l'anode est nulle.

3. La polarisation de la cathode est égale, en valeur absolue, au potentiel du couple en circuit ouvert et de signe négatif.

4. Le courant anodique (c'est-à-dire passant par: le métal le moins noble du couple) résultant est nul.

5. Le courant cathodique résultant est égal au courant total de protection cathodique.
On en arrive à cette conclusion, méconnue mais importante, que, en protection cathodique minimum (et ceci s'étend dans une large mesure à la protection cathodique usuelle), c'est le métal noble - ou cathode - qui consomme la plus grande partie du courant de protection cathodique. Les économies de courant, dans une protection cathodique bien réglée, que l'on peut espérer du revêtement bon isolant du métal le moins noble sont donc moins importantes qu'on le croît généralement.

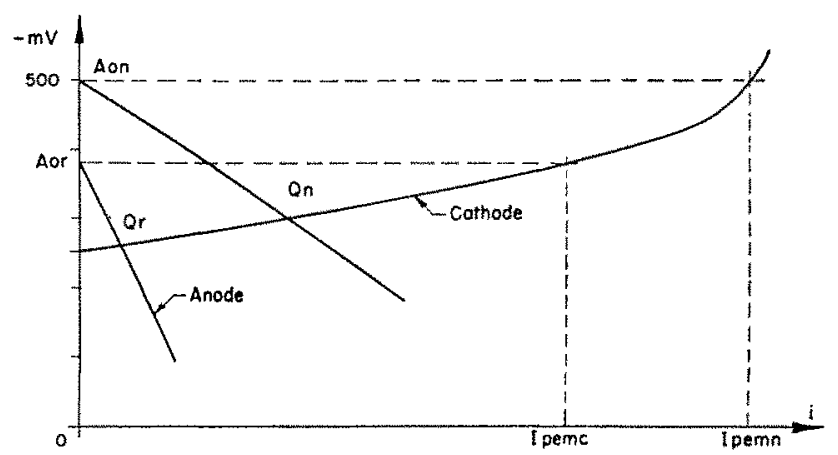

FIa. 10. - Diagramme de polarisation d'une machine peinte :

avec revêtement intact $A$ or $Q r$, avec blessure du revêtement Aon $Q \mathrm{n}$. 
C) Protection cathodique du couple métal nobleacier peint (fig. 10) : c'est le cas le plus complexe.

La peinture, non seulement augmente la résistance du circuit, mais modifie le potentiel de dissolution de l'acier ordinaire. En cas de blessure nous passons du point $Q_{\mathrm{R}}$ de protection cathodique minimum de l'acier revêtu, au point $Q_{n}$ de protection cathodique minimum de l'acier nu.

Nous devons done nous attendre, dans le cas d'un métal revètu, à une certaine instabilité du potentiel de l'anode, ce que nous avons parfaitement observé expérimentalement en Rance, où les plaques se trouvaient assez fréquemment blessées, done à une certaine instabilité du courant de protection cathodique nécessaire, ce qui posera peut-être des problèmes de régulation.

\subsection{3. - VARIATION DE I'ALLURE DES COURBES DE POLARISATION EN FONCTION DE LA VITESSE DE L'ÉLEGTROLYTE :}

Ce phénomène intéressant a été mis en évidence avec netteté par l'équipe Changarnier lors de l'étude des courbes de polarisation de certains métaux.

\section{A) Description du phénomène :}

A vitesse nulle de l'électrolyte, les courbes de certains métaux ont une allure classique, la partie Vo A (fig. 11) correspond à une limitation de

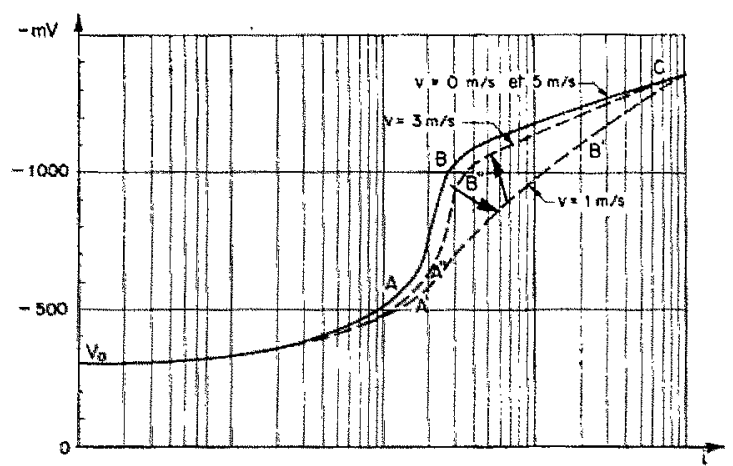

Fic. 11. - Evolution de la zone de polarisation par diffusion ea fonction de la vitesse de l'électrolyle.

la dépolarisation cathodique par la lenteur de réduction de l'oxygène, la partie $A B$ à une limitation de la dépolarisation des cathodes par diffusion trop lente de l'oxygène de la masse de l'électrolyte vers la cathode; la partie BC correspond à une dépolarisation par l'hydrogène ou par d'autres ions. Si on augmente la vitesse du courant d'eau jusque vers $2 \mathrm{~m} / \mathrm{s}$, la partie $A B$, à courant presque constant, se modifie dans le sens d'une augmentation du courant, ce qui se traduit sur le graphique par la partie $A^{\prime} B^{\prime}$.

Si on augmente, au-delà de 2 à $3 \mathrm{~m} / \mathrm{s}$, la vitesse de l'eau, la courbe a tendance à se rapprocher de son allure initiale à vitesse nulle, suivant la loi $\mathrm{A}^{\prime \prime} \mathrm{B}^{\prime \prime}$.

\section{B) Explication classique :}

Akimov [5] a bien noté qu'en fonction de la vitesse de l'électrolyte la corrosion allait en augmentant, puis en diminuant (fig. 12). Enfin elle augmente de nouveau jusqu'au phénomène de cavitation.

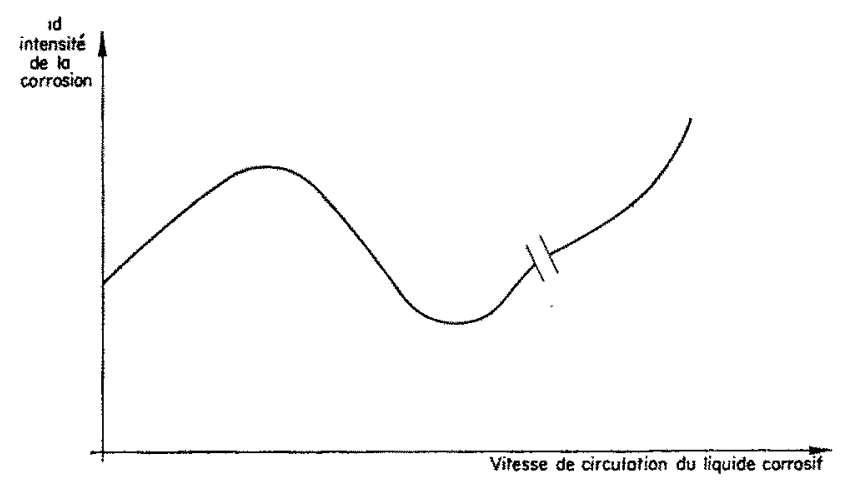

FIG. 12. - Allure générale de la relation entre la vitesse de corrosion et la vitesse de circulation du liquide corosif (Akimov).

Quand l'eau se met en mouvement, elle apporte des particules d'oxygène pour la dépolarisation de la cathode, aidant ainsi le phénomène de diffusion (corrosion croissante); l'eau circulant plus vite, un apport plus important d'oxygène passiverait ce métal (corrosion décroissante) jusqu'à la rupture de tout édifice superficiel au moment de la cavitation (corrosion croissante à nouveau).

\section{C) Explication proposée :}

Cette explication se heurte à plusieurs objections : le métal devrait être marqué par cette passivation et une diminution brutale de la vitesse vers $1 \mathrm{~m} / \mathrm{s}$ devrait donner, dans les premières secondes, une meilleure résistance à la corrosion dans le premier essai : il n'en est rien.

Aux grandes vitesses -5 mètres par seconde par exemple - le comportement des particules d'oxygène vis-à-vis de la couche passivante serait le même que vis-à-vis de la couche de diffusion, coinneidence bien peu probable.

Nous suggérons une nouvelle explication prenant en compte l'existence de la couche laminaire au voisinage de la paroi immobile.

Lors des essais à vitesse lente, l'énergie cinétique des particules d'oxygène de la couche laminaire est faible. 
Ces particules d'oxygène, amenées dans la zone d'aspiration de la diffusion, ne peuvent en ressortir : elles y sont piégées.

Si on augmente la vitesse du courant d'eau, on augmente l'énergie moyenne de turbulence des particules d'oxygène; cette grande énergie cinétique peut vaincre le potentiel d'attraction de la diffusion et fait sortir statistiquement autant de particules d'oxygène qu'il en était rentré. Dès lors, le phénomène se retrouve à peu près identique à ce qu'il était à vitesse nulle, les particules d'oxygène de petite énergie au voisinage immédiat des parois continuent à être drainées par diffusion, la turbulence n'apportant rien parce qu'elle emporte autant.

Cette explication demande des vérifications ex- périmentales el, si elle est confirmée, pourra donner lieu à une thérie quantitative.

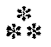

Nous avons donné un apercu très rapide de nos essais de revêtements dont certains autorisent de grands espoirs, de nos essais de métaux sur la machine G.E.M. et dans l'appareil de polarisation: les essais nous apportent des apaisements sur.les possibilités de cohabitation de plusieurs métaux, mais attirent notre attention sur des précautions indispensables..

Je profite de cette occasion pour remercier Mlle Kammerlocher, ma collaboratrice dans ce travail, ainsi que M. Delville et M. Romanovsky, dont les observations nous ont été précieuses.

\section{BIBLIOGRAPHIE}

1. Evans. - Corrosion des métaux $\$ 1951$ et Congress of pure and Applied Chemistry.

*An outline of corrosion mechanisms, including the electro-chemical Theory ». The Corrosion Handbook, edited by H.H. Uhlig, London, Chapman and Hall Ltd.

2. Branchi. - « Contributign expérimentale à la connaissance des phénomènes de réduction cathodique de l'oxygène et de l'eau oxygénée $\gg$. Corrosion et anti-corrosion, Vol. $\mathrm{V}, \mathrm{n}^{\circ} 5$, mai 1957 .

3. Dechavx (G.). - « Essais accélérés de laboratoire, essais au radeau, essais sur bateau des peintures anti-corrosives de carènes $\gg$, p. 3, fig. 4 .

4. Pope (R.). - «Cell Currents and Potentials », Corrosion, Vol. 11. Conférence à la National Association of Corrosion Engineers, N. Y., mars 1951.

5. Akimov. - « La corrosion des métaux », Dunod, 1956

6. Laque et Stewart. - Métaux el Corrosion, $\mathrm{n}^{\circ} 274$, juin 1948.

7. Teel (R. B.). - « Some observations of the potentials of stainless steel in flowing sea water $\gg$. Corrosion, Vol. 8, juillet 1956.

8. Delville (J.). - « 11 rapports sur le comportement des éprouvettes E.D.F. à la station de contrôle de corrosion de Cézembre »(internes à l'E.D.F., non publiés).

9. Dechaux. - « Protection de l'acier de construction. - Principe du peinturage protecteur $\gg$, Chemistry and Industry, 1955, 48, p. 1535-1541.
10. Dechaux. - * Protection, au moyen de peinture, des carèzes en acier, contre les actions destructives de la mer \$. - Peinture, pigments, vernis, Vol. 17 janvier, octobre et novembre 1942.

11. «Steel Structures Painting Manual », Vol. 1 et 2, publiés par le Steel Structures Painting Council, 4400, Fifth Avenue, Pittsburg-13 (Pa), 1955.

12. Garner (James B.), S.S.P.C. - « Steel Structures Painting Manual ». - Vol. 1: \& Quality Control of Paints 》, p. 151, fig. 2.

13. Romanovsky, - Rapports intemes a l'E.D.F., n ${ }^{0 s} 3$ $4,5,6,7,8$

14. Changarnien. - Rapports internes à l'E.D.F., $n^{a *} 1$, $2,3,4,5,6$.

15. «Steel Structure Painting Manual », Vol. 2 : « Paint Systems Guide », tableau IX, p. 209.

16. Devolux (R. P.). - « The painting of Steel vessels for salt wates service $\gg$ S.S.P.M. Vol. 1 , p. 266 et 27, fig. 3 ,

17. MAXNE (J.E.O.), - \& Current views on how paint film prevent corrosion $\gg$, Journal of the oil and colour Chemists Association, Vol. 40, $\mathrm{n}^{\circ} 3$, mars 1957, p. 183 et suivantes.

18. Dans (J.) et Schuster (H.J.). - « Theoretische und electrochemische Grundlagen des Verhaltens von Zinc in Anstrichsystemen $»$, Farbe und Lack, septembre 1957

\section{ISCUSSION}

\section{Plisident : M. Gibnat}

M. le Président remercie vivement MM. Ratri et Sunred et indique que la discussion de la communication aura Jieu ultérieurement, soit dans une séance spéciale, soit au cours d'une prochaine session.

Pour donner à cette discussion tout le développement qu'elle mérite, les membres intéressés sont priés de demander entre temps, a $N$. VANTRors, chef du service des usines marémotrices d'Electricité de France, 44, rue de Lisbonne, à Paris, un exemplaire de la communication de MM. Rath et Surrel et de se faire inscrire pour être invitès à prendre part à la discussion.

M. Hupnen suggère l'étude de la corrosion des métaux à l'état de précontrainte.

Un autre membre voudrait savoir, dans le même esprit, comment se comportent les infrastructures rocheuses.

Suite de la discussion (séance du 22 novembre 1957).
La séance est ouverte à 16 h 50 , sous la présidence de M. Natror, chef de la Division \& Coordination Technique $\gg$ du Service de la Production Hydraulique d'E.D.F.

Après avoir souhaité la bienvenue à tous, M. le Président demande à M. Sunres de bien vouloir rappeler les grandes lignes de l'exposé sous discussion.

Les principaux points de la communieation de MM. RATH et SURrel sont les suivants :

- Les expériences de Saint-Malo sont valables pour un cas particulier de corrosion. L'application de nos conclusions à d'autres cas ne doit se faire sans réflexion critique;

- Etude des revêtements protecteurs des métaux;

- Etude de la résistance à la corrosion des métaux nobles, et de la possibilite de leur assoctation;

-.- Protection cathodique; 
- La théorie de la couche de collage et le cloquage des revêtements;

- L'étude du dioptre métal-eau au point de vue thermodynamique et l'évolution de la polarisation.

La discussion est ouverte à 17 h. 15 .

M. Surner complète son résumé en signalant que les peintures sont poreuses. Au microscope, à un grossissement de 100 , elles peurent présenter l'aspect d'un «feutre » plus ou moins compact, aspect probablement lié à la résistance mécaníque médiocre de beaucoup de revêtements, les peintures vinyliques semblent moins mauvaises à ces points de vue.

M. Dechaux confirme les observations de M. Surrel et, pour concrétiser le phénomène, fait passer des échantillons prélevés sur des bouées à Saint-Malo en expliquant que ce phénomène de décollement de couches encore saines se retrouve à Marseille comme à SaintMalo. Il fait circuler ane photo en couleur où l'on distingue nettement la couche qui se décolle.

M. Surrel fait encore quelques remarques :

- La cloque est entourée d'une zone de mauvaise adhérence;

- Le décollement a lieu souvent, entre le wash primer et la première couche de primer;

- Des études dirigées par M. Changarnier laissent entrevoir la possibilité d'améliorer l'adhérence des peintures.

M. le Président demande si, le phénomène de libération d'hydrogène lourd qui se produit notamment pour les aciers Martin, n'est pas une raison de décollement si la couche barrière est rigoureusement étanche.

M. Dechaux pense plutôt qu'il se produit un décollement parce qu'il y a arrivée d'eau et' d'oxygène au contact du métal. Il n'y a pas en général de dégagement d'hydrogène dans la corrosion marine. Généralement, les cloques sont le plus souvent remplies de liquỉde et l'on constate que les ions $\mathrm{OH}$ y sont en plus grand nombre que dans l'eau de mer : on a une solution très basique.

M. Surner pense qu'il ne faut cependant pas écarter le phénomène de dégagement d'hydrogène par le métal comme l'une des causes mineures de la naissance des cloques.

Pour répondre à la question de M. LabBens sur les procédés métallurgiques susceptibles d'améliorer la tenue de la peinture, M. le Président indique qu'une solution déjà employée, consiste à dégazer le métal par un traitement thermique : il s'agit d'une chauffe prolongée à $250^{\circ}$ qui provoque le dégagement d'hydrogène et ne mo. difie pas les caractéristiques mécaniques du métal.

M. Charbonnier voit une contradiction entre la porosité de la couche de protection et le fait que l'hydrogène reste emprisonné.

M. le Président estime que pour que l'hydrogène lourd libéré par l'acier soit la cause du décollement de la couche, il faut que celle-ci soit imperméable (cas des antifrictions).

M. Dechaux fait alor's la différence entre le cas des métaux ou couches métalliques qui sont imperméables et celui des corps organiques poreux à l'oxygène et à l'eau (Mayne)

M. le Président signale qu'avec un chromage sur l'acier il n'y a pas de phénomène de décollement, le ehrome galvanique étant un revêtement absolument poreux. II n'y a pas possibilité de mise en pression de l'hydrogène lourd, comme sous l'antifriction.

M. Dechaux ajoute que l'on est en présence d'un phém nomène d'osmose : la concentration étant différente d'ui côté et de l'autre de la paroi, il y a gonflement. Il peul y avoir également un phénomène d'électroosmose.

M. Surrel pense qu'il n'y a pas incompatibilité entre tout ce qui vient d'être dif : précisément, dans son exposć, il considère la couche de collage intacte, comme perméable; les autres couches de peinture étant imbibées d'eau sous forme d'un réseau de gouttelettes reliées par des mierocanaux (voir J.E.o. Mayne).

Ce réseau arrive au voisinage immédiat de la couche de collage mais ne semble pas en affecter l'imperméabilité jusqu'à ce que la couche soit blessée. Pour l'hydrogène, cette couche peut être assez antidiffusante quand elle existe; l'hydrogène pourrait alors la soulever; dès lors elle n'existe plus, tout devient perméable.

M. le Président fait remarquer qu'actuellement la technique est de diminuer l'épaisseur des conches et d'en augmenter le nombre afin d'augmenter l'étanchéité transversale en diminuant la porosité.

A la question posée par M. Bruzaud-Grille: «si la couche d'accrochage se décolle par suite d'un dégagement d'hydrogène gazeux en provenance de l'acier lui-même, au lieu de rechereher une couche absolument imperméable, ne peut-on rechercher une peinture semi imperméable, laissant passer de l'hydrogène, mais continuant à assurer une protection suffisante du mćtal? 》 M. Surrel répond qu'une telle peinture pourrait effectivement avoir de l'intérêt pour d'autres raisons, mais il répète que l'hydrogène lui semble une cause mineure de cloquage.

M. Mary demande où reste le wash primer: sur le métal on sur la cloque; s'il reste sur le métal, n'est-ce pas une raison pour penser que le dégagement d'hydrogène n'intervient pas dans la formation de la cloque? M. Vantroys pense que cette conclusion n'est peut-être pas obligatoire dans le cas général et que l'on ne peut pas écarter a priori l'éventualité de rencontrer un wash primer perméable sous une couche supérieure imperméable.

Selon M. Surrel, on retrouve assez sonvent le wash primer sur l'acier mais, en accord avec M. VANTroys, cela ne lui permet pas de conclure que le phénomène de "collage» le plus important n'a pas lieu au dioptre acier/revêtement.

M. Surrel espère qu'un chercheur se penchera sur cette question de l'aspect microphysique du collage, ce qui, à sa connaissance, n'a pas étè fait jusqu'à présent.

A la demande d'un participant, il rappelle qu'un nonreau procédé d'application de revêtement consiste à pulvériser la peinture à chaud, sous forte pression interne, sans l'aide d'air comme fluide palvérisateur, le revêtement est atomisé à la sortie de la buse, car il contient un solvant léger liquide tant qu’il est sous pression, qui explose à la pression atmosphérique. Il a ćté envisagé également, une projection sous charge électrostatique.

M. le Président mentionne que cette technique électrostatique a déjà été développée chez Pechiney pour to soufrage et qu'elle est utilisée pour la peinture. On construit en France des générateurs de peinture à 100 uu $150 \mathrm{KV}$. Ce procédé, très utilisé (peinture cellulosique), donne de bons résultats et ne laisse aucun déchet; seulement 4 ou $5 \%$ s'en vont sous forme de brouillard.

M. Dechaux ajoute que l'on peint ainsi, en atelier, des objets de toutes sortes mais ce procédé lui paraît difficile à appliquer pour les navires ou les chantiers hydro. électriques.

M. le Président pense que cela n'est pas impossible gràce aux générateurs électrostatiques portatifs.

M. Dechatux ne pense pas que les couches de vinylique posées sous potentiel électrostatique ou pulvérisées sans air soient plus homogenes que les autres: toute évaporation d'un solvant ou d'un autre laisse des trous an milieu de tourbillons hexagonaux de solifluxion (phenomène de Benard) et seule, la multiplication des conches peut probablement éliminer la coincidence de ces trous.

M. Davio revient à l'hypothèse du dégagement d'hydrogène issu de l'acier et demande comment on peut concilier ce dégagement qu'on serait en droit de supposer homogène sur toute la surface du métal protégé, avec lo caractère très local des cloques. Il faudrait supposer que le phénomène de décollement intervient sur une très grande surface à la fois, ou que lhydrogène dégagé se trouve canalisé à un point de moindre résistance de lat couche de peinture. 
M. le Président répond que le dégagement est régulier mais il $\mathrm{y}$ a localisation de l'hydrogene en un point, oú il s'accumule et monte en pression. If $y$ a analogie avec les «flocons».

Passant à la question de la tenue des métuux, M. le Président rappelle qu'un grand nombre de nuances de bronze el d'aciers inoxydables ont été essayćs, entre autres les aciers 20/12 qui sont les plus fortement alliés.

M. SURrel indique que la résistance à la corrosion est fonction de la teneur en chrome et, pour une moindre part en nickel.

M. MarX demande si le déplacement d'ion $H$ vers un métal noble cathodique ne risque pas de fragiliser ce métal.

M. Surrel répond que la dépolarisation cathodique sous ces faibles potentiels s'effectue par réduction de l'atome d'oxygène dissous en ion $\mathrm{OH}^{-}$. La réduction

$$
\mathrm{H}^{+}+\mathrm{e}^{-} \rightarrow \mathrm{H}^{\prime \prime}
$$

est thermodynamiquement impossible pour la généralité des couples étudiés (voir texte de la conférence complète).

A la question de M. MANY sur la valeur du potentiel pour laquelle l'ion $H$ apparait, $M$. le Président signale ru'il faut atteindre les tensions de l'ordre de celle de l'electrolyse de l'eau, éest-à-dire de l'ordre de $1000 \mathrm{mV}$.

M. LeGrand donne alors quelques chifires :

- L'association d'un acier ordinaire et d'un acier inoxydable forme un couple dont la différence de potentiel à vide est de l'ordre de $200 \mathrm{mV}$, Le potentiel de l'acier Martin A 37 par rapport à l'eau de mer est de l'ordre de $600 \mathrm{mV}$, mesuré avec l'électrode au calomel.

En fait, le taux de corrosion dépend du débit de ce couple: ce débit est réglé par la résistance totale du circuit qui comprend la résistance externe ou électronique (résistance de contact des métaux avec le milieu) af la résistance interne ou résistance ionique.

La virulence de la corrosion dépend ensuite de la densité du courant.

M. le Président fait ressortir l'idée essentielle qui se dégage des travaux de M. Changarnier : Il s'attache a la notion de courant et délaisse la notion de potentiel de dissolution : dans ces piles souvent en court-circuit que sont les couples intermétalliques les phénomènes de polarisation réduisent le courant et s'il y a peu de courant, il y a peu de transport de métal, done peu de corrosion. N'ayant pas les documents nécessaires pour répondre a $M$. Carousy sur le elasscment des différents métaux et les qualités des bronzes inox, M. Legrand indique cependant que le courant de couple bronze-acier ordinaire, est plus important que le courant de couple acicr inoxydable-acier ordinaire, et M. SuRxel ajoute que la différence de potentiel en circuit ouvert n'est pas plus forte pour l'association bronze-acier ordinaire que pour l'asso. ciation acier inox-acier ordinaire. C'est l'intérêt de la notion de courant de couple qui décèle des dangers inapparents à l'examen des potentiels de dissolution.

A l'aide du schéma ci-après, M. Sunfex donne de plus amples explications.

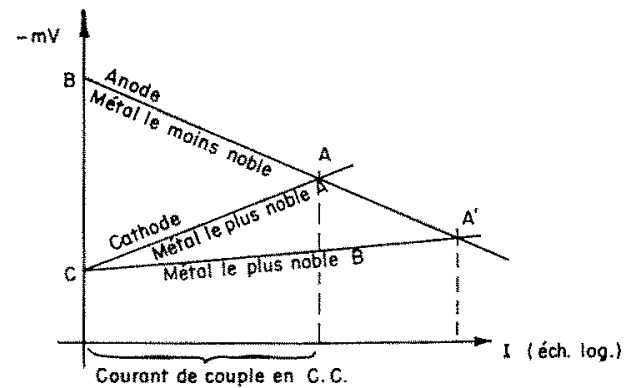

A l'origine, en cireuit ouvert, la différence de potentiel a la valeur maximum : quand le courant passe il $y$ a polarisation à la fois de l'anode et de la cathode; la différence de potentiel entre métaux décroit, se rappro- che de zéro; le courant de couple, critère de corrosion, n'est pas nul pour antant.

La figure montre comment, avec la mème différence de potentiel cie dissolution at $i=0$, le metal $B$ peut donner une corrosion plus intense que le métal $\mathrm{A}$.

L'équipe de M. Chancamina étudie done et classe les métaux non pius d’après la différence de potentiel, mais d'après l'intensité de courant qui circule quand on établit le court-circuit entre les deux métaux qui sont alor: nu même potentiel (courant correspondant au point A). De là on arrive à la notion de stabilité. On dit que l'as. sociation de deux métaux est trés stable quand leur courant de couple est inféricur à 10 microampères par cm².

M. le Président conclut que ce sont les classements par les courants qui sont les plus natureis en milienx agressifs.

M. Kamerlochen fait remarquer l'importance de l'état de surface sur l'attaque ou le développement de la corrosion des métaux nobles.

M. SURREL confirme qu'on a observé sur des machines G.E.M. que de deux pales du mème métal, inégalement polies, c'est la mieux polie qui résiste le mieux. Les petites criques de la pale non polic sont l'objet d'inclusions, centres de micropiles locales de corrosion. Si par polissage, on détruit ces différences de potentiel, on supprime cette cause de corrosion.

M. Surnel répond affirmativement à la question de M. Labbens sur la sensibilité relative aux défauts de sur. face de l'acier à $13 \%$ de chrome et d'autres aciers plus riches en chrome: il indique diatre part que lacier $17 / 4 / 4$ se place à ce point de vue entre l'acier de $13 \%$ de chrome et les aciers plus riches en chrome.

M. De Saint-Vaulay demande si l'on peut comparer les attaques faites par l'eau de mer arec les dissolutions ducs à l'eau distillée chaude et d'une grande résistivité électrique (ce qui semble diminuer l'attaque électrochimi(que).

M. Sunrer pense que non; 11 répète que tontes les expériences de Saint-Malo ne sont valables que pour l'eau de mer et qu'aucun résultat ne peut être transposé à l'eau douce, distillée on non.

M. le Président ajoute alors que les essais ont déte menés parallèlement en cau de mer artificielle ef en eau de mer vivante. Les résultats étaient différents: il semble qu'il $y$ a progressivité de la corrosion lorsqu'on passe de l'eau artificielle à l'eau de mer naturelle.

M. Dechaux ajoute qu'il y a lieu de tenir compte d'un cerlain nombre de facteurs, entre autres de la vilesse de la température, du $\mathrm{pH}$ de l'eau et des sels dissous.

M. David intervient ensuite, et signale que lattaque d'une tôle d'acier inoxydable (3\% de chrome, $18,8 \%$ de nickel) se porte sur les defauts de fonderie. On obtient donc dans les alliages précités un métal noble actif (dans les piqûres), et un métal passif. Comme il existe une différence de potentiel entre ces parties, on doit s'at. tendre à une autopropagation de la corrosion, seulement le sens du courant ne pourra être précisé, le défaut pouvant être anode ou cathode.

M. Surnes indique qu'aucune expérience n'a été faite à ce sujet, mais l'explication de M. David lui parait probable.

Un des participants fait les dernières remarques ci après:

- Si l'on arrivait à trouver une protection cathodique à régime constant, le problème serait résolu; or pour des groupes immergés dans l'eau de mer, on a affaire à des salinités différentes et à des températures différentes, il faudra une protection cathodique qui s'adapte a chaque régime.

M. Legnand répond que, si le seuil de protection cathodique est bien délimité, par contre, au dessus de ce seuil, on a une marge assez grande pour englober les phénomènes de ce genre: température et salinite différentes.

M. le Président clôt la séance en félicitant MM. RATH et Sunkel de leur excellent travail.

La seance est levée a 18 h. 17 . 